\title{
Type synthesis of freedom and constraint elements for design of flexure mechanisms
}

\author{
H.-J. Su ${ }^{1}$ and C. Yue ${ }^{2}$ \\ ${ }^{1}$ Department of Mechanical and Aerospace Engineering, Ohio State University, Columbus, Ohio, 43210, USA \\ ${ }^{2}$ Department of Mechanical Engineering, University of Maryland Baltimore County, Baltimore, \\ Maryland, 21250, USA \\ Correspondence to: H.-J. Su (su.298@osu.edu)
}

Received: 3 March 2013 - Accepted: 19 April 2013 - Published: 17 July 2013

\begin{abstract}
In this paper, we present the type synthesis of freedom and constraint elements for design of general flexure mechanisms. As an important step in the conceptual design stage, the goal of type synthesis is to qualitatively determine the topology or connectivity of flexure elements and rigid bodies in a mechanism. The synthesis procedure presented here is based on a recently emerging screw theory based approach for flexure mechanisms. We first categorize a list of commonly used atomic flexure primitives including blades, wires, notches and bellow springs etc. We then derive their twist and wrench matrices that mathematically represent their freedom and constraint spaces. The synthesis procedure rigorously follows screw algebra. Freedom elements including R-joints and P-joints are defined as basic motion elements that allow a single rotation or a single translation. By using parallel structures of these flexure primitives, eleven designs of R-joints and eight designs of P-joints are systematically synthesized. As a duality, constraint elements including P-constraints and R-constraints remove a single translation or rotation. In contract to freedom elements, we synthesized serial chains of flexure primitives and obtained six designs of P-constraints and three designs of R-constraints. These freedom and constraint elements form a catalogue of basic building blocks for designing more complex flexure mechanisms. At last we utilize four design examples to demonstrate how to synthesize hybrid structures with serial and parallel combination of these elements.
\end{abstract}

\section{Introduction}

Compliant mechanisms (Howell, 2001) or flexure mechanisms (Smith, 2000; Smith and Chetwynd, 1992), formed by a set of rigid bodies connected with compliant elements, produce a defined motion through elastic deformation of their compliant elements. They are widely used in various precision instruments and machines such as nano-manipulators Culpepper and Anderson (2004), nano-positioners (Chen and Culpepper, 2006; Brouwer et al., 2010; Yao et al., 2008; Dong et al., 2008) and precision manufacturing machines (Varadarajan and Culpepper, 2007a,b).

The design of flexure mechanisms has been an ad hoc process that heavily relies on designers' experience and intuition that are typically built up over years of training. One approach often used in precision engineering community is called the constraint-based approach (Blanding, 1999; Hale, 1999; Awtar and Slocum, 2007). In a recent conference tutorial by Henein (2011), the author enumerated a list of flexure bearing designs based on their degree of freedom (DOF), Grubler mobility and degree of hyperstaticity (DOH). Many authors have attempted to systemize the constraint based approach. Recently this approach has been further formalized into the Freedom and Constraint Topology (FACT) framework (Hopkins, 2007a; Hopkins and Culpepper, 2010a,b). Essentially the constraint-based design approach and the FACT approach are mathematically equivalent to screw theory (Ball, 1998; Hunt, 1978; Phillips, 1984, 1990; Davidson and Hunt, 2004) that has been widely used in kinematics community for various problems such as type synthesis of parallel mechanisms (Kong and Gosselin, 2010) and mobility analysis of rigid body mechanisms (Huang et al., 2008). 
In recognizing this intrinsic connection between the constraint based approach and screw theory, a series of work ( $\mathrm{Su}$ et al., 2009; Su and Tari, 2010, 2011; Hopkins and Culpepper, 2010c; Yu et al., 2010; Su, 2011) on screw theory based approach for type synthesis and analysis of flexure mechanisms have been done. This approach is completely based on screw algebra (Dai and Jones, 2001, 2003), which can be easily implemented in computer programs for truly automating the design process of flexure mechanisms, especially the conceptual design stage. However currently these work have been focusing on mechanisms with relatively simple topologies or mobility analysis (rather than synthesis). The type synthesis of general flexure mechanisms for any specified mobility is still yet to be done.

In this paper, we present a systematic methodology based on screw theory for the type synthesis of general flexure mechanisms with serial, parallel or hybrid (combination of serial and parallel) topologies. First, a list of commonly used flexure primitives is studied. Then they are used to build basic freedom and constraint elements. At last these freedom and constraint elements are used for constructing more complex flexure mechanisms.

\section{Screw theory overview}

In this section, we first review basic concepts of screw theory as a background preparation for the following sections.

\subsection{Twists and wrenches}

A flexure mechanism subject to a general load represented by a wrench $\hat{W}$ undergoes an instantaneous motion represented by a twist $\hat{T}$. From the freedom and constraint point of view, twists represent allowable motions while wrenches represent forbidden motions. Both twist $\hat{T}$ and wrench $\hat{W}$ are 6 by 1 column vectors, written as

$$
\begin{aligned}
& \hat{T}=\left\{\begin{array}{l}
\mathbf{\Omega} \\
\mathbf{V}
\end{array}\right\}=\left\{\begin{array}{c}
\mathbf{\Omega} \\
\mathbf{c} \times \mathbf{\Omega}+p \boldsymbol{\Omega}
\end{array}\right\}, \\
& \hat{W}=\left\{\begin{array}{c}
\mathbf{F} \\
\mathbf{M}
\end{array}\right\}=\left\{\begin{array}{c}
\mathbf{F} \\
\mathbf{c} \times \mathbf{F}+q \mathbf{F}
\end{array}\right\},
\end{aligned}
$$

where $p$ and $q$ are called pitches of twist and wrenches. And $\hat{T}$ and $\hat{W}$ satisfy the so called reciprocal condition:

$\hat{T} \circ \hat{W}=\mathbf{\Omega} \cdot \mathbf{M}+\mathbf{V} \cdot \mathbf{F}=0$.

A general rotational or translational freedom respectively corresponds to a twist with zero or infinite pitch, written as

$\hat{T}_{R}=\left\{\begin{array}{c}\mathbf{\Omega} \\ \mathbf{c} \times \mathbf{\Omega}\end{array}\right\}, \quad \hat{T}_{P}=\left\{\begin{array}{c}\mathbf{0} \\ \mathbf{V}\end{array}\right\}$.

Similarly a general rotational or translation constraint removes a rotation or translation along a particular direction. They respectively correspond to a wrench with infinite or zero pitch, written as

$\hat{W}_{R}=\left\{\begin{array}{c}\mathbf{0} \\ \mathbf{M}\end{array}\right\}, \quad \hat{W}_{P}=\left\{\begin{array}{c}\mathbf{F} \\ \mathbf{c} \times \mathbf{F}\end{array}\right\}$

For convenience, we define six principal twists as the rotation and translations about the three coordinate axes,

$$
\begin{aligned}
\hat{R}_{x} & =\left(\begin{array}{llllll}
1 & 0 & 0 & 0 & 0 & 0
\end{array}\right)^{T} \\
\hat{R}_{y} & =\left(\begin{array}{llllll}
0 & 1 & 0 & 0 & 0 & 0
\end{array}\right)^{T} \\
\hat{R}_{z} & =\left(\begin{array}{llllll}
0 & 0 & 1 & 0 & 0 & 0
\end{array}\right)^{T} \\
\hat{P}_{x} & =\left(\begin{array}{llllll}
0 & 0 & 0 & 1 & 0 & 0
\end{array}\right)^{T} \\
\hat{P}_{y} & =\left(\begin{array}{llllll}
0 & 0 & 0 & 0 & 1 & 0
\end{array}\right)^{T} \\
\hat{P}_{z} & =\left(\begin{array}{llllll}
0 & 0 & 0 & 0 & 0 & 1
\end{array}\right)^{T}
\end{aligned}
$$

Similarly, we also define six principal wrenches as the rotational and translational constraint about the three coordinate axes

$$
\begin{aligned}
\hat{F}_{x} & =\left(\begin{array}{llllll}
1 & 0 & 0 & 0 & 0 & 0
\end{array}\right)^{T} \\
\hat{F}_{y} & =\left(\begin{array}{llllll}
0 & 1 & 0 & 0 & 0 & 0
\end{array}\right)^{T} \\
\hat{F}_{z} & =\left(\begin{array}{llllll}
0 & 0 & 1 & 0 & 0 & 0
\end{array}\right)^{T} \\
\hat{M}_{x} & =\left(\begin{array}{llllll}
0 & 0 & 0 & 1 & 0 & 0
\end{array}\right)^{T} \\
\hat{M}_{y} & =\left(\begin{array}{llllll}
0 & 0 & 0 & 0 & 1 & 0
\end{array}\right)^{T} \\
\hat{M}_{z} & =\left(\begin{array}{llllll}
0 & 0 & 0 & 0 & 0 & 1
\end{array}\right)^{T}
\end{aligned}
$$

\subsection{Coordinate transformation of twists and wrenches}

The coordinate transformation of a twist or wrench is calculated as

$\hat{T}^{\prime}=[\mathbf{A d}] \hat{T}, \quad \hat{W}^{\prime}=[\mathbf{A d}] \hat{W}$,

where $\hat{T}, \hat{W}$ and $\hat{T}^{\prime}, \hat{W}^{\prime}$ correspond to the twist and the wrench before and after the transformation. And [Ad] is the so-called $6 \times 6$ adjoint matrix, written as

$[\mathbf{A d}]=\left[\begin{array}{cc}R & 0 \\ D R & R\end{array}\right]$

where $[\mathbf{R}]$ is a 3 by 3 rotation matrix and $[\mathbf{D}]$ is the 3 by 3 skew-symmetric matrix defined by the translational vector $\boldsymbol{d}=\left(d_{x}, d_{y}, d_{z}\right)^{T}$. They have the form

$[\mathbf{R}]=\left[\begin{array}{lll}\mathbf{x} & \mathbf{y} & \mathbf{z}\end{array}\right], \quad[\mathbf{D}]=\left[\begin{array}{ccc}0 & -d_{z} & d_{y} \\ d_{z} & 0 & -d_{x} \\ -d_{y} & d_{x} & 0\end{array}\right]$

When the coordinate transformation is applied to a principal screw (twist or wrench), the resultant screw will along 
the axes of the new coordinate system, e.g.

$$
\begin{aligned}
& \hat{R}_{x}^{\prime}=[\mathbf{A d}] \hat{R}_{x}=\left\{\begin{array}{c}
\mathbf{x} \\
\mathbf{d} \times \mathbf{x}
\end{array}\right\} \\
& \hat{P}_{x}^{\prime}=[\mathbf{A d}] \hat{P}_{x}=\left\{\begin{array}{l}
\mathbf{0} \\
\mathbf{x}
\end{array}\right\}
\end{aligned}
$$

\section{Flexure primitives}

A flexure primitive is defined as an "atomic" flexure mechanism that consists of only one flexure element and zero intermediate body. They cannot be further divided into substructures. Recently Hopkins (2012) presented three commonly used flexures and their freedom and constraint spaces using FACT approach. In this section, we first categorize a more comprehensive set of commonly used flexure primitives and derive their freedom and constraint spaces. Then we will discuss a general synthesis methodology for constructing serial and parallel kinematic chains of these flexure primitives.

\subsection{Commonly used flexure primitives}

According to the mobility or the rank of their twist system, we can categorize the most commonly used flexure primitives as shown in Table 1.

1. A notch hinge, denoted by symbol "R", allows a rotation about the centerline and constraints other motions. The shape of the cross section may be circular, elliptical, hyperbolic etc. Meanwhile short beams, living hinges that have one dimension significantly smaller than others can function as a notch hinge. A split tube which is a tube sliced along its longitudinal direction also allows a single rotation about its axis. If we define the axis of the $\mathrm{R}$-joint to be the $\mathrm{z}$-axis, its twist and wrench matrices can be written as

$$
\left[T_{r}\right]=\left[\hat{R}_{z}\right], \quad\left[W_{r}\right]=\left[\begin{array}{lllll}
\hat{F}_{x} & \hat{F}_{y} & \hat{F}_{z} & \hat{M}_{x} & \hat{M}_{y}
\end{array}\right]
$$

2. A spherical notch or short wires/rod, denoted by "S", allows three rotations and constrains three translations. Kinematically it is equivalent to a serial chain of three $\mathrm{R}$-joints, i.e. $\mathrm{S}=3 \mathrm{R}$. Their twist and wrench matrices are

$$
\left[T_{s}\right]=\left[\begin{array}{lll}
\hat{R}_{x} & \hat{R}_{y} & \hat{R}_{z}
\end{array}\right], \quad\left[W_{s}\right]=\left[\begin{array}{lll}
\hat{F}_{x} & \hat{F}_{y} & \hat{F}_{z}
\end{array}\right]
$$

3. Blade/sheet flexures also called leaf springs, denoted by "B", allow two rotations and one translation, i.e. $\mathrm{B}=2 R-P$. The rotational symmetric cylinders and disc rings also have the same freedom and constraint spaces. As shown in Table 1, we define the normal of the blade as the $y$-axis and the longitudinal direction being the $\mathrm{x}$-axis. The two rotations are about two in-plane axes $(x, z)$ due to beam torsion and beam bending respectively. And the translation is along the normal direction $y$, caused by beam bending. From the constraint point of view, a blade removes one rotation and two translations. Their twist and wrench matrices are

$$
\left[T_{b}\right]=\left[\begin{array}{lll}
\hat{R}_{x} & \hat{R}_{z} & \hat{P}_{y}
\end{array}\right], \quad\left[W_{b}\right]=\left[\begin{array}{lll}
\hat{F}_{x} & \hat{F}_{z} & \hat{M}_{y}
\end{array}\right]
$$

4. A long wire/rod flexure, denoted by "W", removes the translation along its axis and allows the other five motions. A corner blade (a folded sheet) also provides a single constraint along its fold line. Their freedom space is equivalent to three R-joints and two P-joints, i.e. $\mathrm{W}=3 \mathrm{R}-2 \mathrm{P}$. Mathematically its corresponding twist and wrench matrices are

$$
\left[T_{w}\right]=\left[\begin{array}{lllll}
\hat{R}_{x} & \hat{R}_{y} & \hat{R}_{z} & \hat{P}_{y} & \hat{P}_{z}
\end{array}\right], \quad\left[W_{w}\right]=\left[\hat{F}_{x}\right]
$$

5. And lastly, a bellow spring, denoted by " $\mathrm{B}_{\mathrm{s}}$ ", removes a single rotation along its axis with a freedom space denoted by $\mathrm{B}_{\mathrm{s}}=2 \mathrm{R}-3 \mathrm{P}$. Its twist and wrench matrices are

$$
\left[T_{\mathrm{bs}}\right]=\left[\begin{array}{lllll}
\hat{R}_{x} & \hat{R}_{y} & \hat{P}_{x} & \hat{P}_{y} & \hat{P}_{z}
\end{array}\right], \quad\left[W_{\mathrm{bs}}\right]=\left[\hat{M}_{z}\right]
$$

Table 1 summarizes the aforementioned flexure primitives and their freedom space and twist and wrench matrices. These primitives are basic building blocks for constructing more complex flexure systems.

\subsection{Serial chains of flexure primitives}

The freedom space of a rigid body represents all of its allowable motion in space. For any given flexure element or building block, its freedom space is a $f$ system that is represented by a twist matrix [T] formed by $f$ independent twists, written as

$[\mathbf{T}]=\left[\begin{array}{lll}\hat{T}_{1} & \hat{T}_{2} & \cdots \hat{T}_{f}\end{array}\right]$

When a mechanism is formed by $m$ flexure elements or building blocks that are connected in serial, its twist matrix (freedom space) can be obtained by combining the twist matrix of each building block column-wise, mathematically written as

$[\mathbf{T}]=\left[\begin{array}{llll}A d_{1} T_{1} & A d_{2} T_{2} & \cdots & A d_{m} T_{m}\end{array}\right]$

where $\left[A d_{j}\right]$ represent the coordinate transformation from the $j$-th building block to the functional stage, defined in (9). This formulation has been previously presented by $\mathrm{Su}$ (2011). Also see similar work by Hopkins (2007b); Hopkins and Culpepper (2011).

By applying a column-wise reduction, we can easily obtain a set of independent $r=\operatorname{rank}(T)$ twists that forms the basis of the freedom space. $r$ is also called the mobility or degree-of-freedom of the mechanism. Any set of $r$ independent twists called basis can span the entire freedom space. 
Table 1. The motion and constraint spaces of commonly used flexure primitives.

\begin{tabular}{l}
{$[\mathbf{T}]$} \\
{$[\mathbf{W}]$} \\
\hline
\end{tabular}

The transformation from one basis to another is done by a linear operation to the twist matrix.

A freedom space can be spanned by different sets of basis twists. This can be very useful in design practices. For instance, consider a freedom space formed by rotations about two parallel axes. The twist matrix of these two rotations is

$[\mathbf{T}]=\left[\begin{array}{ll}\hat{T}_{R_{1}} & \hat{T}_{R_{2}}\end{array}\right]=\left[\begin{array}{cc}\boldsymbol{\Omega} & \boldsymbol{\Omega} \\ \mathbf{c}_{1} \times \mathbf{\Omega} & \mathbf{c}_{2} \times \mathbf{\Omega}\end{array}\right]$

Subtracting the second column from the first one yields

$[\mathbf{T}] \doteq\left[\begin{array}{cc}\mathbf{\Omega} & \mathbf{0} \\ \mathbf{c}_{1} \times \mathbf{\Omega} & \left(\mathbf{c}_{2}-\mathbf{c}_{1}\right) \times \mathbf{\Omega}\end{array}\right]$

where $\doteq$ represents a column-wise linear operation. Note the second column of the above matrix represents a translation along the direction normal to both the rotation axis and the line $\mathbf{c}_{1} \mathbf{c}_{2}$. Basically this means that a serial chain of two rotations is equivalent to a serial chain of a rotation and a translation. See the top part of Fig. 1.

Similarly, a serial chain of three (non-coplanar) parallel rotations is equivalent to a rotation plus two orthogonal trans- lations. They are formulated in screws as

$$
\begin{aligned}
{[\mathbf{T}] } & =\left[\begin{array}{ll}
\hat{T}_{R_{1}} \hat{T}_{R_{2}} & \hat{T}_{R_{3}}
\end{array}\right]=\left[\begin{array}{ccc}
\boldsymbol{\Omega} & \boldsymbol{\Omega} & \boldsymbol{\Omega} \\
\mathbf{c}_{1} \times \mathbf{\Omega} & \mathbf{c}_{2} \times \mathbf{\Omega} & \mathbf{c}_{3} \times \mathbf{\Omega}
\end{array}\right] \\
& \doteq\left[\begin{array}{ccc}
\mathbf{\Omega} & \mathbf{0} & \mathbf{0} \\
\mathbf{c}_{1} \times \mathbf{\Omega} & \left(\mathbf{c}_{2}-\mathbf{c}_{1}\right) \times \mathbf{\Omega} & \left(\mathbf{c}_{3}-\mathbf{c}_{1}\right) \times \mathbf{\Omega}
\end{array}\right],
\end{aligned}
$$

where the second and third columns represent translations. See the bottom part of Fig. 1 for illustration of this case.

\subsection{Parallel chains of flexure primitives}

The constraint space of a rigid body represents all the forbidden motions of the body subject to a constraint arrangement. In screw theory, a constraint space can be represented by a wrench matrix $[\mathbf{W}]$ combined by $c$ independent wrenches, written as

$$
[\mathbf{W}]=\left[\begin{array}{llll}
\hat{W}_{1} & \hat{W}_{2} & \cdots & \hat{W}_{c}
\end{array}\right]
$$

The constraint space of a flexure mechanism formed by $m$ building blocks connected in parallel is given by a wrench matrix that is obtained by assembling the wrench matrix of each building block, written as

$[\mathbf{W}]=\left[\begin{array}{llll}A d_{1} W_{1} & A d_{2} W_{2} & \cdots & A d_{m} W_{m}\end{array}\right]$ 

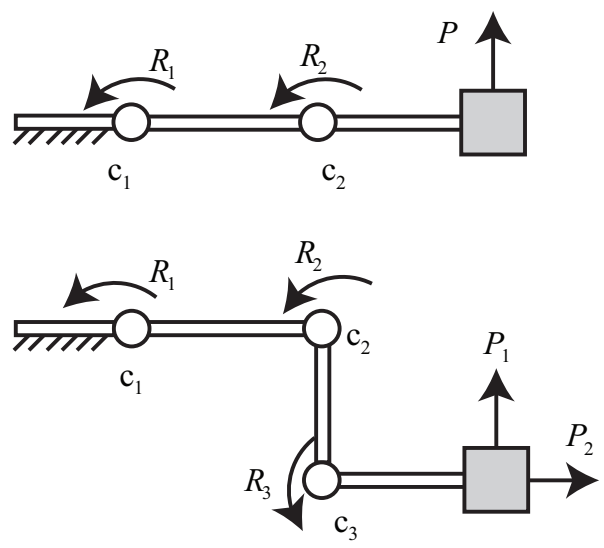

Figure 1. Equivalent freedom spaces. (Top) A serial chain of two parallel rotations produces a translation. (Bottom) A serial chain of three (non-coplanar) parallel rotations produce two translation.

Again, matrices $\left[\mathbf{A d}_{j}\right]$ represent the coordinate transformation from $j$-th building block to the functional stage.

Similar to serial chains of flexures, the constraint space of two parallel translational constraints assembled in parallel (Fig. 2) is represented by two force wrenches with identical direction $\mathbf{F}$

$[\mathbf{W}]=\left[\begin{array}{ll}\hat{W}_{P_{1}} & \hat{W}_{P_{2}}\end{array}\right]=\left[\begin{array}{cc}\mathbf{F} & \mathbf{F} \\ \mathbf{c}_{1} \times \mathbf{F} & \mathbf{c}_{2} \times \mathbf{F}\end{array}\right]$

Subtracting the second column from the first one yields

$[\mathbf{W}]=\left[\begin{array}{cc}\mathbf{F} & \mathbf{0} \\ \mathbf{c}_{1} \times \mathbf{F} & \left(\mathbf{c}_{2}-\mathbf{c}_{1}\right) \times \mathbf{F}\end{array}\right]$

Note the second column of the above matrix represents a rotational constraint along the direction perpendicular to both the constraint axis $\mathbf{F}$ and the line $\mathbf{c}_{1} \mathbf{c}_{2}$. Basically this says that a parallel chain of two translational constraints removes one rotation and one translation.

And a parallel chain of three (non-coplanar) parallel translational constraints removes two rotations and one translation. This is illustrated with the following mathematical formulation

$$
\begin{array}{r}
{[\mathbf{W}]=\left[\hat{W}_{P_{1}} \hat{W}_{P_{2}} \hat{W}_{P_{3}}\right]=\left[\begin{array}{c}
\mathbf{F F F} \\
\mathbf{c}_{1} \times \mathbf{F} \mathbf{c}_{2} \times \mathbf{F} \mathbf{c}_{3} \times \mathbf{F}
\end{array}\right]} \\
\dot{=}\left[\begin{array}{c}
\mathbf{F 0 0} \\
\mathbf{c}_{1} \times \mathbf{F}\left(\mathbf{c}_{2}-\mathbf{c}_{1}\right) \times \mathbf{F}\left(\mathbf{c}_{3}-\mathbf{c}_{1}\right) \times \mathbf{F}
\end{array}\right]
\end{array}
$$

\section{Synthesis of freedom elements}

In this section, we use flexure primitives listed in Table 1 to design flexure joints that have only one DOF, rotational $(\mathrm{R})$ or translational $(\mathrm{P})$. To design freedom elements, we use parallel chains of primitives to remove unwanted freedoms.
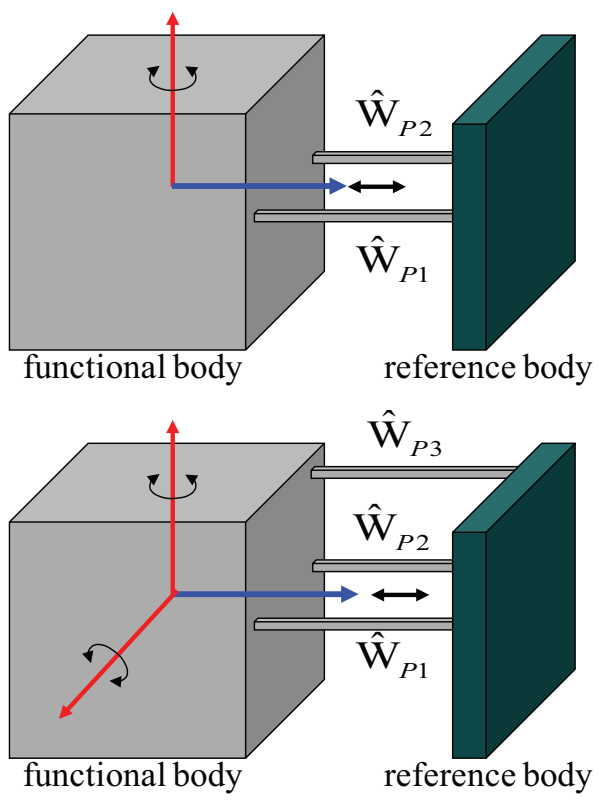

Figure 2. Equivalent constraint spaces. (Top) A parallel chain of two parallel translational constraints exerts a translational plus a rotational constraint to the functional body. (Bottom) A parallel chain of three (non-coplanar) parallel translational constraints exerts a translation plus two rotational constraints. The arrows represent the removed motions.

\subsection{Synthesis of R-joints $\hat{T}_{R}$}

The freedom space of an R-joint is given by $\hat{T}_{R}$ shown in (4). Without loss of generality, we assume the axis of R-joint through the origin, i.e. $\mathbf{c}=(0,0,0)^{T}$. This gives us

$\hat{T}_{R}=\left\{\begin{array}{c}\mathbf{\Omega} \\ \mathbf{0}\end{array}\right\}$

Our goal is to remove the other five unwanted motions: two rotations and three translations. Since we consider parallel structures of at least two limbs and each limb must apply 1-5 constraints to the functional body, primitive " $R$ " (notch hinge) cannot be used in the design as it is indeed a R-joint. This leads us total 11 possible combinations with the other four primitives $\mathrm{B}, \mathrm{S}, \mathrm{W}$ and $\mathrm{B}_{\mathrm{s}}$. Among them, cases 1-5 use no bellow springs and cases $6-11$ use at least one bellow spring.

\section{Use flexure primitives B and W only}

The first three cases concerns designs with primitives $\mathrm{B}$ and $\mathrm{W}$ only: $2 \mathrm{~B}, \mathrm{~B}-2 \mathrm{~W}, 5 \mathrm{~W}$. These are the most commonly used designs.

\subsubsection{Case 1:2B}

The synthesis with two parallel blades (2B) is described as the following. As shown in Fig. 3, let us denote the 


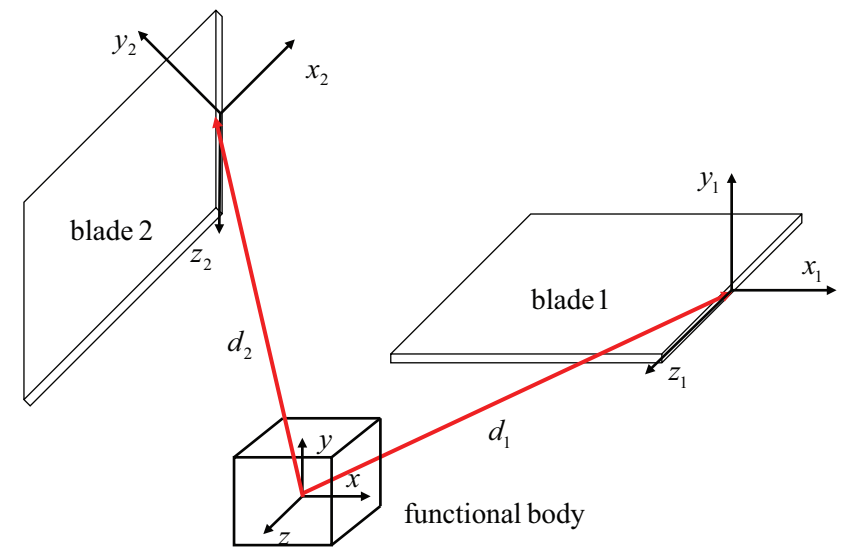

Figure 3. Synthesis of R-joints with a parallel connection of two blades. The two blades are rigidly connected to the functional body.

coordinate transformation of two blades relative to the functional body by $\left[\mathbf{A d}_{1}\right]$ and $\left[\mathbf{A d}_{2}\right]$ which are the six by six adjoint matrices with the form of (9). By applying the coordinate transformation to both blades, their wrench matrices are calculated by $(10,11)$ as

$\left[\mathbf{W}_{i}\right]=\left[\mathbf{A d}_{i}\right]\left[\mathbf{W}_{b}\right]=\left[\begin{array}{ccc}\mathbf{x}_{i} & \mathbf{z}_{i} & \mathbf{0} \\ \boldsymbol{d}_{i} \times \mathbf{x}_{i} & \boldsymbol{d}_{i} \times \mathbf{z}_{i} & \mathbf{y}_{i}\end{array}\right], i=1,2$,

where $\left[\mathbf{W}_{b}\right]$ is the wrench matrix of blade written in (14). And vectors $\mathbf{x}_{i}, \mathbf{y}_{i}, \mathbf{z}_{i}, \boldsymbol{d}_{i}$ denote the orientation and position of $i$-th blade.

Requiring the reciprocity of the $\left[\mathbf{W}_{i}\right]$ with $\hat{T}_{R}$ yields the necessary condonations regarding to the orientation and position of the blades

$$
\begin{array}{rlll}
\boldsymbol{\Omega} \cdot\left(\boldsymbol{d}_{i} \times \mathbf{x}_{i}\right) & =0, & \Longrightarrow & \mathbf{x}_{i} \cdot\left(\boldsymbol{d}_{i} \times \mathbf{\Omega}\right)=0 \\
\mathbf{\Omega} \cdot\left(\boldsymbol{d}_{i} \times \mathbf{z}_{i}\right) & =0, & \Longrightarrow & \mathbf{z}_{i} \cdot\left(\boldsymbol{d}_{i} \times \mathbf{\Omega}\right)=0 \\
\mathbf{\Omega} \cdot \mathbf{y}_{i} & =0 . & &
\end{array}
$$

Simplifying the first two conditions yields

$\boldsymbol{d}_{i} \cdot \mathbf{y}_{i}=0, \quad \boldsymbol{\Omega} \cdot \mathbf{y}_{i}=0$.

The first equality is interpreted as that the translation $\boldsymbol{d}_{i}$ must in the blade plane $\mathbf{x}_{i} \mathbf{z}_{i}$. The second condition means that $\mathbf{y}_{i}$, the normal direction of the blade, must be perpendicular to the axis of the R-joint $(\boldsymbol{\Omega})$. If $\boldsymbol{\Omega}=(1,0,0)^{T}, \mathbf{y}_{i}$ must be in the plane $y z$ of the functional body. Picking any two independent directions in the $y z$ plane leads us a solution, e.g.

$\mathbf{y}_{1}=(0,1,0)^{T}, \quad \mathbf{y}_{2}=(0,0,1)^{T}$

Once $\mathbf{y}_{i}$ is determined. The axes of $\mathbf{x}_{i}$ and $\mathbf{z}_{i}$ are chosen arbitrarily as long as the two blades are not co-planar (redundant).

Depending on how $\mathbf{x}_{i}$ and $\mathbf{z}_{i}$ are chosen for each blade, we can obtain three possible designs. If $\mathbf{z}_{1}=\mathbf{z}_{2}=(1,0,0)^{T}$, we have a design with both blades subject to bending, shown in Fig. 4a. This is the most often used flexure hinge design. If $\mathbf{x}_{1}=\mathbf{z}_{2}=(1,0,0)^{T}$, we have a design with blade 1 subject to twisting and blade 2 subject to bending, Fig. $4 \mathrm{~b}$. If $\mathbf{x}_{1}=$ $\mathbf{x}_{2}=(1,0,0)^{T}$, we have a design with both blades subject to twisting, Fig. 4c.

\subsubsection{Case 2: B-2W}

This design evolves from the case $2 \mathrm{~B}$. Since a blade allows two rotations and one translation, we just need to remove one rotation and one translation with two wires. We have shown in (27) that two parallel constraints remove one translation and one rotation. Therefore, replacing one blade of the 2B design with two parallel wires yields a B-2W design, shown in Fig. 4d.

\subsubsection{Case 3: 5W}

In order to obtain a hinge design with five wires, we simply replace the blade of the $\mathrm{B}-2 \mathrm{~W}$ design with three co-planar wires. This is due to the fact that a blade is equivalent to three co-planar wires. See Fig. 4e. An alternative synthesis procedure using screw algebra for the case five wires can be found in Su and Tari (2010).

\section{Use flexure primitives S, B, W}

Now we consider designs using primitives $\mathrm{S}, \mathrm{B}, \mathrm{W}$ with at least one S-joint. There are three possible combinations: $2 \mathrm{~S}$, S-2W, B-S.

However the case B-S is not qualified as a R-joint for the following reasons. Without loss of generality, we let the coordinate system align with the local coordinate system of the blade shown in Table 1. And we denote the position of Sjoint by $\boldsymbol{d}=\left(d_{x}, d_{y}, d_{z}\right)^{T}$. Therefore the wrench matrix of the blade and the $\mathrm{S}$-joint are

$\left[\mathbf{W}_{b}\right]=\left[\begin{array}{lll}\hat{R}_{x} & \hat{R}_{z} & \hat{P}_{y}\end{array}\right],\left[\mathbf{W}_{s}^{\prime}\right]=\left[\begin{array}{ccc}\boldsymbol{i} & \boldsymbol{j} & \boldsymbol{k} \\ \boldsymbol{d} \times \boldsymbol{i} & \boldsymbol{d} \times \boldsymbol{j} & \boldsymbol{d} \times \boldsymbol{k}\end{array}\right]$

where vectors $\boldsymbol{i}, \boldsymbol{j}, \boldsymbol{k}$ are unit vectors along three coordinate axes. And $\left[\mathbf{W}_{s}^{\prime}\right]$ is the wrench matrix with an appropriate coordinate transformation, i.e. $\left[\mathbf{W}_{s}^{\prime}\right]=[\mathbf{A d}]\left[\mathbf{W}_{s}\right]$. We write the wrench matrix of the parallel structure B-S and apply a linear operation to obtain

$\left[\begin{array}{l}\mathbf{W}_{\mathrm{bs}}\end{array}\right]=\left[\begin{array}{ll}\mathbf{W}_{b} & \mathbf{W}_{s}^{\prime}\end{array}\right]=\left[\begin{array}{cccccc}1 & 0 & 0 & 1 & 0 & 0 \\ 0 & 0 & 0 & 0 & 1 & 0 \\ 0 & 1 & 0 & 0 & 0 & 1 \\ 0 & 0 & 0 & 0 & -d_{z} & d_{y} \\ 0 & 0 & 1 & d_{z} & 0 & -d_{x} \\ 0 & 0 & 0 & -d_{y} & d_{x} & 0\end{array}\right]$ 


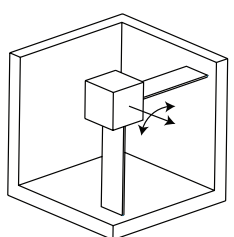

(a)

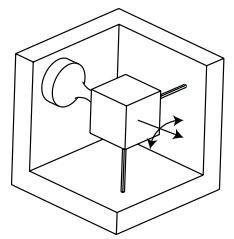

(g)

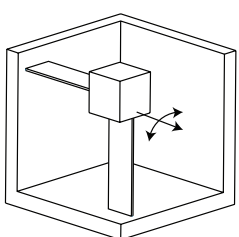

(b)

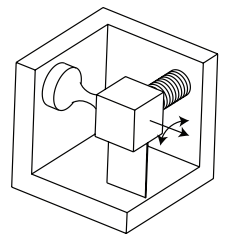

(h)

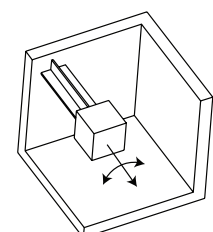

(c)

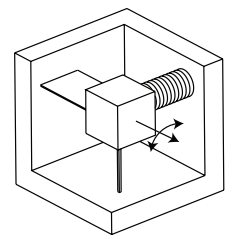

(i)

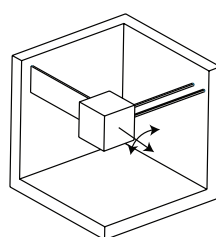

(d)

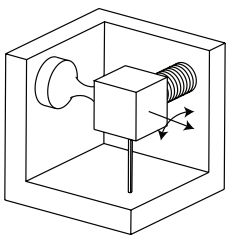

(j)

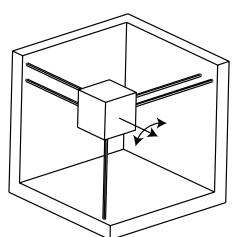

(e)

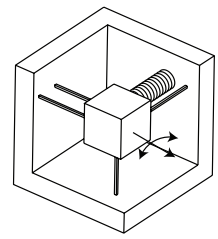

(k)

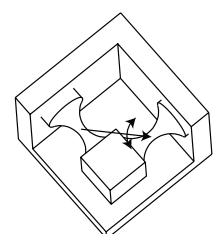

(f)

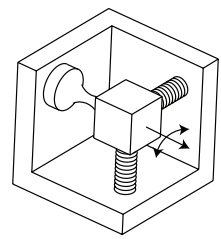

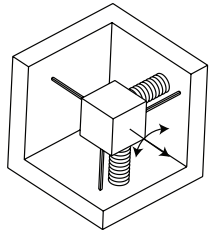

(m)

Figure 4. Various designs of R-joints with flexure primitives: B, W, S and $\mathrm{B}_{\mathrm{s}}$. (a-c) case 1 with two blades (BB). (d) case 2 with one blade and two wires $(\mathrm{B}-2 \mathrm{~W})$. (e) case 3 with five wires $(5 \mathrm{~W})$. (f) case 4 with two spherical notches (2S). (g) case 5 with one spherical notch with two wires $(\mathrm{S}-2 \mathrm{~W})$. (h) case 6 with one bellow spring, one blade and one spherical notch $\left(\mathrm{B}_{\mathrm{s}}-\mathrm{B}-\mathrm{S}\right)$. (i) case 7 with one bellow spring, one blade and one wire $\left(\mathrm{B}_{\mathrm{s}}-\mathrm{B}-\mathrm{W}\right)$. (j) case 8 with one bellow spring, one spherical notch and one wire $\left(\mathrm{B}_{\mathrm{s}}-\mathrm{S}-\mathrm{W}\right)$. (k) case 9 with one bellow spring and four wires $\left(\mathrm{B}_{\mathrm{s}}-4 \mathrm{~W}\right)$. (l) case 10 with two bellow springs and one spherical notch $\left(2 \mathrm{~B}_{\mathrm{s}}-\mathrm{S}\right)$. (m) case 11 with two bellow springs and three wires $\left(2 \mathrm{~B}_{\mathrm{s}}-3 \mathrm{~W}\right)$. The double arrow arcs represent the rotation allowed by flexure R-joints. The box represents the functional body.

$$
\doteq\left[\begin{array}{cccccc}
1 & 0 & 0 & 0 & 0 & 0 \\
0 & 0 & 0 & 0 & 1 & 0 \\
0 & 1 & 0 & 0 & 0 & 0 \\
0 & 0 & 0 & 0 & -d_{z} & d_{y} \\
0 & 0 & 1 & 0 & 0 & 0 \\
0 & 0 & 0 & -d_{y} & d_{x} & 0
\end{array}\right]
$$

From Eq. (38), we can draw two conclusions. (1) If $d_{y} \neq 0$, $\left[\mathbf{W}_{\mathrm{bs}}\right]$ has a full rank which means that the body is fully constrained by this structure. (2) If $d_{y}=0$, i.e. the $S$-joint is on the blade plane, $\left[\mathbf{W}_{\mathrm{bs}}\right]$ has a rank of 2 since the 4 th and 6 th column would be zeros. As a matter of fact, its reciprocal freedom space is formed by the two rotations allowed by the blade flexure. Therefore, parallel structures of B-S cannot form a R-joint design.

\subsubsection{Case 4:2S}

The $2 \mathrm{~S}$ case is trivial. When two S-joints are connected in parallel, this results in a single rotation about the line connecting to their joint centers, Fig. 4f.

\subsubsection{Case 5: S-2W}

The case $\mathrm{S}-2 \mathrm{~W}$ is synthesized as the following. The S-joint itself removes three translations. We just need to remove two additional rotations with two wire flexures. By using a wire parallel to y-axis with a non-zero offset $\boldsymbol{d}=(d, 0,0)^{T}$, the rotation $\hat{R}_{z}$ is removed. Similarly, a wire parallel to $\mathrm{z}$-axis with a non-zero offset $\boldsymbol{d}=(d, 0,0)^{T}$ removes the rotation $\hat{R}_{y}$. Mathematically, the wrench matrix of S-joint and the two wires are
$\left[\mathbf{W}_{s}\right]=\left[\begin{array}{ccc}1 & 0 & 0 \\ 0 & 1 & 0 \\ 0 & 0 & 1 \\ 0 & 0 & 0 \\ 0 & 0 & 0 \\ 0 & 0 & 0\end{array}\right], \quad\left[\mathbf{W}_{2 \mathrm{w}}\right]=\left[\begin{array}{cc}0 & 0 \\ 1 & 0 \\ 0 & 1 \\ 0 & 0 \\ 0 & -d \\ d & 0\end{array}\right]$

The constraint space of S- $2 \mathbf{W}$ is obtained by combining $\left[\mathbf{W}_{s}\right]$ and $\left[\mathbf{W}_{2 \mathrm{w}}\right]$, to which we apply a column-wise reduction and obtain

$\left[\begin{array}{ll}\mathbf{W}_{s-2 \mathrm{~W}}\end{array}\right]=\left[\begin{array}{ll}\mathbf{W}_{s} & \mathbf{W}_{2 \mathrm{~W}}\end{array}\right]=\left[\begin{array}{ccccc}1 & 0 & 0 & 0 & 0 \\ 0 & 1 & 0 & 0 & 0 \\ 0 & 0 & 1 & 0 & 0 \\ 0 & 0 & 0 & 0 & 0 \\ 0 & 0 & 0 & 0 & -d \\ 0 & 0 & 0 & d & 0\end{array}\right]$

Obviously its reciprocal twist matrix is $\hat{R}_{x}$ which corresponds to a single rotation about the $\mathrm{x}$-axis. This design is shown in Fig. $4 \mathrm{~g}$.

\section{Use flexure primitives $B_{s}, S, B, W$}

At last, we synthesize the design cases with at least one bellow spring. If one $B_{s}$ flexure is used, there are four possible design cases: $\mathrm{B}_{\mathrm{s}}-\mathrm{B}-\mathrm{S}, \mathrm{B}_{\mathrm{s}}-\mathrm{B}-\mathrm{W}, \mathrm{B}_{\mathrm{s}}-\mathrm{S}-\mathrm{W}, \mathrm{B}_{\mathrm{s}}-4 \mathrm{~W}$. And if two bellow springs are used, there are two additional combinations: $2 \mathrm{~B}_{\mathrm{s}}-\mathrm{S}$ and $2 \mathrm{~B}_{\mathrm{s}}-3 \mathrm{~W}$. Note $2 \mathrm{~B}_{\mathrm{s}}-\mathrm{B}$ is not possible as $\mathrm{B}$ removes one rotation and $2 \mathrm{~B}_{\mathrm{S}}$ remove another two rotation which results in a freedom space with no rotation. Let us discuss these six cases in the following. 


\subsubsection{Case 6: $\mathrm{B}_{\mathrm{s}}-\mathrm{B}-\mathrm{S}$ :}

From Eq. (38), we have concluded that a B-S parallel structure may result in a freedom space of two rotations if the center of S-joint is on the blade plane. Now we just need to use a bellow spring $B_{s}$ to remove one rotation in order to yield a freedom space of only one rotation, hence a hinge design in Fig. 4h.

\subsubsection{Case 7: $\mathrm{B}_{\mathrm{s}}-\mathrm{B}-\mathrm{W}$ :}

As we know one blade has a freedom space of 2R-P. To obtain a single rotation, we can use a bellow spring to remove one rotation and a wire to remove the translation. See this design in Fig. 4i.

\subsubsection{Case 8: Bs-S-W:}

The S-joint has a freedom space of $3 R$. To obtain a single rotation, we can use a bellow spring to remove one rotation then use a wire offset to the center of the S-joint to remove the second rotation. This results a design shown in Fig. 4j.

\subsubsection{Case 9: $B_{\mathbb{s}}-4 W$}

To synthesize this case, we first use three wires aligning the three coordinate axes to remove three translations. This leaves us three rotations. We then remove two more rotations by two more wires. By Eq. (26), we know that two parallel constraints remove one additional rotation. Therefore we let a fourth wire be parallel to one of the three wires aforementioned. This fourth wire removes a rotation about the normal line to the plan formed by the two parallel wires. At last, a bellow spring is used to remove another rotation. This results a hinge design shown in Fig. 4k.

\subsubsection{Cases 10: 2Bs $-\mathrm{S}$}

The synthesis of $2 \mathrm{~B}_{\mathrm{s}}-\mathrm{S}$ case is simple. The $\mathrm{S}$-joint removes three translations and two bellow springs remove two of the three rotations, Fig. 41.

\subsubsection{Cases 11:2 $\mathrm{B}_{\mathrm{s}}-3 \mathrm{~W}$}

This case is also trivial. First the three wires aligning the three coordinate axes remove three translations. And we then use two bellow springs remove two rotations. This design is shown in Fig. 4m.

\subsection{Synthesis of P-Joints $\hat{T}_{P}$}

Now let us synthesize P-joints with flexure primitives listed in Table 1. The freedom space of an P-joint is given by a single twist

$\hat{T}_{P}=\left\{\begin{array}{l}\mathbf{0} \\ \mathbf{V}\end{array}\right\}$ where vector $\mathbf{V}$ represent the translational direction of P-joint.

We are interested in simple parallel structures with at least two limbs that remove three rotations and two translations. And each limb must apply at least one constraint and allow at least one translational motion for the functional body. Therefore primitives " $\mathrm{R}$ " and " $\mathrm{S}$ " are not qualified as they allow no translation. This leaves three possible primitives: $\mathrm{B}, \mathrm{W}$ and $\mathrm{B}_{\mathrm{s}}$ from which we can have eight possible cases. Three of them use no bellow springs and the other five use at least one bellow spring.

\section{Designs with flexures B, W only}

Considering only flexure $\mathrm{B}$ and $\mathrm{W}$, we have three cases: $2 \mathrm{~B}$, B-2W and $5 \mathrm{~W}$.

\subsubsection{Case 1: 2B}

For designs with two blades, the reciprocity conditions of $\hat{T}_{P}$ in (41) with the wrench matrices $\left[\mathbf{W}_{i}\right]$ in (30) yields

$\mathbf{V} \cdot \mathbf{x}_{i}=0, \quad \mathbf{V} \cdot \mathbf{z}_{i}=0, \quad i=1,2$

from which we conclude that $\mathbf{V}$ must be parallel to both $\mathbf{y}_{i}$ (normal of the blade plane). This means that two blades must be parallel with the normal of the blade plane along the axis of P-joint. This is the well known parallel sheet design of P-joints shown in Fig. 5a.

\subsubsection{Case 2: B-2W}

The design with one blade and two wires can be easily obtained by replacing one blade with two wires that are parallel to the blade plane. Figure $5 \mathrm{~b}$.

\subsubsection{Case 3: 5W}

This design is obtained from the B-2W design by replacing one blade with three co-planar wires and the other one with two wires. This case can also be computationally synthesized with screw theory. Assume the P-joint is along the $x$ direction, i.e. $\mathbf{V}=(1,0,0)^{T}$. First of all, compute the reciprocal wrench matrix of $\hat{P}$ using linear algebra as

$$
[\mathbf{W}]=\left[\begin{array}{lllll}
0 & 0 & 0 & 0 & 0 \\
1 & 0 & 0 & 0 & 0 \\
0 & 1 & 0 & 0 & 0 \\
0 & 0 & 1 & 0 & 0 \\
0 & 0 & 0 & 1 & 0 \\
0 & 0 & 0 & 0 & 1
\end{array}\right]
$$


We then apply a column-wise linear operation to this wrench matrix to obtain

$$
\left[\mathbf{W}^{\prime}\right]=\left[\begin{array}{lllll}
0 & 0 & 0 & 0 & 0 \\
1 & 1 & 0 & 0 & 1 \\
0 & 0 & 1 & 1 & 0 \\
0 & 0 & 0 & 0 & 1 \\
0 & 0 & 0 & 1 & 0 \\
0 & 1 & 0 & 0 & 0
\end{array}\right]
$$

Each column of $\left[\mathbf{W}^{\prime}\right]$ is a force wrench which can be realized with a long wire flexure $(\mathrm{W})$. The $5 \mathrm{~W}$ design is shown in Fig. 5b. See the work by Su and Tari (2010) for an alternative synthesis procedure for this case.

\section{Designs with flexures $B, W$ and $B_{s}$}

There are five cases with at least one bellow spring: $\mathrm{B}_{\mathrm{s}}-\mathrm{B}$ $\mathrm{W}, \mathrm{B}_{\mathrm{s}}-4 \mathrm{~W}, 2 \mathrm{~B}_{\mathrm{s}}-\mathrm{B}, 2 \mathrm{~B}_{\mathrm{s}}-3 \mathrm{~W}, 3 \mathrm{~B}_{\mathrm{s}}-2 \mathrm{~W}$. We discuss each in the following.

\subsubsection{Case 4: $B_{s}-B-W$}

A blade B has a freedom space of 2R-P. To obtain a P-joint design, we must remove the two rotations. First we use a wire flexure with axis being parallel and has a non-zero distance to the blade plane to remove one rotation. The second rotation is removed by a bellow spring flexure. See Fig. 5 d.

\subsubsection{Case 5: $B_{\mathrm{S}}-4 \mathrm{~W}$}

This design can be obtained from the case $\mathrm{B}_{\mathrm{s}}-\mathrm{B}-\mathrm{W}$ by replacing the blade with three co-planar wires. A computational way to synthesize this case is applying an alternative linear operation to the wrench matrix $[\mathbf{W}]$ in (43). This time we would like to have four force wrenches and one couple wrench. The new wrench matrix is written as

$\left[\mathbf{W}^{\prime \prime}\right]=\left[\begin{array}{lllll}0 & 0 & 0 & 0 & 0 \\ 1 & 1 & 0 & 0 & 1 \\ 0 & 0 & 1 & 0 & 0 \\ 0 & 0 & 0 & 0 & 1 \\ 0 & 0 & 0 & 1 & 0 \\ 0 & 1 & 0 & 0 & 0\end{array}\right]$

of which the fourth column is a couple wrench and the other four are force wrenches. Realizing each force wrench with a wire and the couple wrench with a bellow spring yields the design of $B_{s}-4 W$. See Fig. 5e.

\subsubsection{Case 6: 2B $\mathrm{B}_{\mathrm{s}}-\mathrm{B}$}

This case is trivial since the two bellow springs remove the two rotations of the blade. This leaves a freedom space with a single translation, hence a P-joint design shown in Fig. 5f.

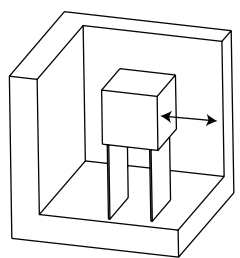

(a)

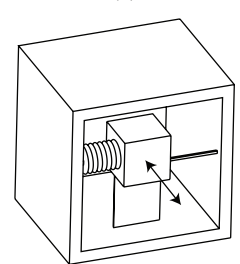

(d)

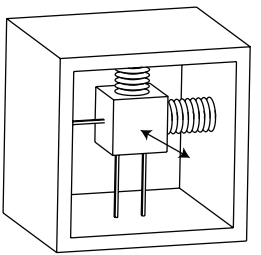

(g)

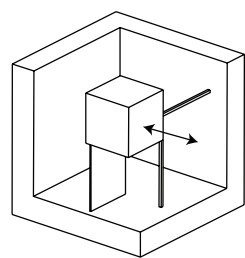

(b)

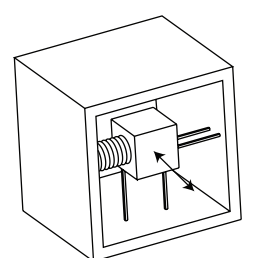

(e)

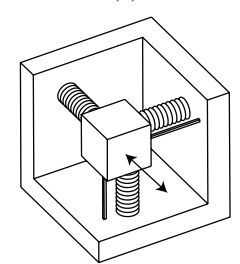

(h)

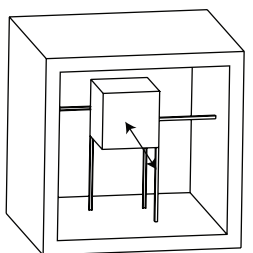

(c)

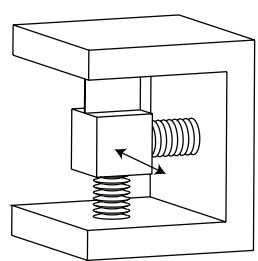

(f)
Figure 5. Various designs of P-joints with parallel structures of flexure primitives: $\mathrm{B}, \mathrm{W}, \mathrm{B}_{\mathrm{s}}$. (a) case 1 with two blades (2B). (b) case 2 with one blade and two wires (B-2W). (c) case 3 with five wires. (d) case 4 with one bellow spring, one blade and one wire $\left(\mathrm{B}_{\mathrm{s}}-\mathrm{B}-\mathrm{W}\right)$. (e) case 5 with one bellow spring and four wires $\left(B_{s}-4 W\right)$. (f) case 6 with two bellow springs and one blade $\left(2 B_{s}-\right.$ B). (g) case 7 with two bellow springs and three wires $\left(2 \mathrm{~B}_{\mathrm{s}}-3 \mathrm{~W}\right)$. (h) case 8 with three bellow springs and two wires $\left(3 \mathrm{~B}_{\mathrm{s}}-2 \mathrm{~W}\right)$. The arrowed lines indicate the direction of translation. The box represents the functional body.

\subsubsection{Case 7:2 $\mathrm{B}_{\mathrm{s}}-3 \mathrm{~W}$}

This case evolves from case $2 \mathrm{~B}_{\mathrm{s}}-\mathrm{B}$ by replacing the blade with three co-planar wires. See Fig. 5g.

\subsubsection{Case 8: 3B-2W}

For this case, the 3 bellow springs remove three translations and two wires remove two translations. This design is shown in Fig. 5 h.

\section{Synthesis of constraint elements}

As a duality to the design of freedom elements, in this section, we design basic constraint elements $\hat{W}_{R}$ and $\hat{W}_{P}$ which remove one rotation and one translation respectively. These constraint elements are most often used in designing parallel structures for removing a particular set of motions. The basis approach is to use a serial chain of flexure primitives listed in Table 1 to construct the complementary freedom space of the constraint element to be synthesized. 


\subsection{Synthesis of P-constraints $\hat{W}_{P}$}

A P-constraint element removes a translational freedom of the functional body while allows all other motions. The constraint space of the translational constraint is given by a pure force given in (5).Without loss of generality, we assume the axis of translational constraint through the origin of the coordinate system, i.e. $\mathbf{c}=(0,0,0)^{T}$. That is

$\hat{W}_{P}=\left\{\begin{array}{l}\mathbf{F} \\ \mathbf{0}\end{array}\right\}$

whose complementary freedom space consists of three rotations plus two translations, i.e. 3R-2P.

To synthesize the translational constraint $\hat{W}_{P}$, we consider serial chains of at least two flexure primitives to make up the complementary freedom space of $\hat{W}_{P}$. We do not include the long wire (W) flexure in the design as itself is indeed a translational constraint. Also bellow springs $\left(\mathrm{B}_{\mathrm{s}}\right)$ are not qualified either as they allow three translations. As a result, only three types of primitives S, B and R are considered in design. Based on the degree of freedom, there are six possible combinations: S-S, S-B, S-2R, B-B, B-2R and 5R. However these primitives must satisfy some restriction on their relative orientation and position. In what follows, we derive the geometric conditions for each case.

\subsubsection{Case 1: S-S}

By intuition, we know that a serial chain of two S-joints removes one translation along the line connecting the center of the joints. Here let us give a mathematical proof with screw theory. Without loss of generality, we assume one S-joint being at the origin of the coordinate system and the other one being at a distance of $d$ along $\mathrm{X}$-axis, i.e. $\boldsymbol{d}=(d, 0,0)^{T}$. The twist matrices of these two blades are

$\left[\mathbf{T}_{1}\right]=\left[\begin{array}{ccc}i & j & k \\ 0 & 0 & 0\end{array}\right], \quad\left[\mathbf{T}_{2}\right]=\left[\begin{array}{ccc}i & j & k \\ d \times i & d \times j & d \times k\end{array}\right]$

By the formulation for serial structures in Eq.(18), the twist matrix of the $\mathrm{S}-\mathrm{S}$ chain is written as

$$
[\mathbf{T}]=\left[\begin{array}{ll}
\mathbf{T}_{1} & \mathbf{T}_{2}
\end{array}\right]=\left[\begin{array}{cccccc}
i & j & k & i & j & k \\
0 & 0 & 0 & d \times i & d \times j & d \times k
\end{array}\right]
$$

Substituting $\boldsymbol{d}=(d, 0,0)^{T}$ and subtracting the last three columns from the first three columns yield

$$
\left[\mathbf{T}^{\prime}\right]=\left[\begin{array}{cccccc}
\boldsymbol{i} & \boldsymbol{j} & \boldsymbol{k} & \mathbf{0} & \mathbf{0} & \mathbf{0} \\
\mathbf{0} & \mathbf{0} & \mathbf{0} & \mathbf{0} & d \boldsymbol{k} & -d \boldsymbol{j}
\end{array}\right]
$$

which indicates three rotations and two translation along $y-$ and $\mathrm{z}$-axes, i.e. the translation along $\mathrm{x}$-axis is removed. See Fig. 7a. The arrow line represents the removed translation.

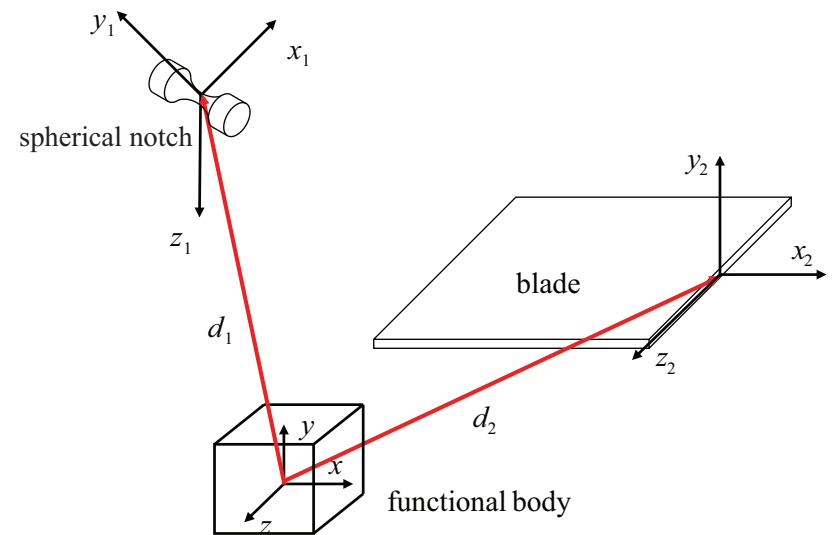

Figure 6. Synthesis of P-constraint with a serial chain of one blade (B) and one spherical notch (S). The blade is connected with the spherical notch via an intermediate body (not drawn)

\subsubsection{Case 2: S-B}

Figure 6 shows a serial chain of S and B flexures. Applying a general coordinate transformation to the twist matrices of the blade and the spherical notch in $(13,14)$ yields

$$
\begin{aligned}
& {\left[\mathbf{T}_{s}\right]=\left[\begin{array}{ccc}
\mathbf{x}_{1} & \mathbf{y}_{1} & \mathbf{z}_{1} \\
\boldsymbol{d}_{1} \times \mathbf{x}_{1} & \boldsymbol{d}_{1} \times \mathbf{y}_{1} & \boldsymbol{d}_{1} \times \mathbf{z}_{1}
\end{array}\right],} \\
& {\left[\mathbf{T}_{b}\right]=\left[\begin{array}{ccc}
\mathbf{x}_{2} & \mathbf{z}_{2} & \mathbf{0} \\
\boldsymbol{d}_{2} \times \mathbf{x}_{2} & \boldsymbol{d}_{2} \times \mathbf{z}_{2} & \mathbf{y}_{2}
\end{array}\right]}
\end{aligned}
$$

Since both freedom spaces are complementary to the prescribed translational constraint, $\hat{W}_{P}$ in (46) is reciprocal to both $\left[\mathbf{T}_{s}\right]$ and $\left[\mathbf{T}_{b}\right]$. The reciprocity condition of S-joint leads to

$$
\begin{array}{lll}
\mathbf{F} \cdot\left(\boldsymbol{d}_{1} \times \mathbf{x}_{1}\right)=0 & \Longrightarrow & \mathbf{x}_{1} \cdot\left(\mathbf{F} \times \boldsymbol{d}_{1}\right)=0 \\
\mathbf{F} \cdot\left(\boldsymbol{d}_{1} \times \mathbf{y}_{1}\right)=0 & \Longrightarrow & \mathbf{y}_{1} \cdot\left(\mathbf{F} \times \boldsymbol{d}_{1}\right)=0 \\
\mathbf{F} \cdot\left(\boldsymbol{d}_{1} \times \mathbf{z}_{1}\right)=0 & \Longrightarrow & \mathbf{z}_{1} \cdot\left(\mathbf{F} \times \boldsymbol{d}_{1}\right)=0
\end{array}
$$

which are reduced to

$\mathbf{F} \times \boldsymbol{d}_{1}=\mathbf{0}, \quad \Longrightarrow \quad \boldsymbol{d}_{1}=d_{1} \mathbf{F}$

This implies that the position of the S-joint must be along the direction of $\mathbf{F}$.

And the reciprocity requirement for the blade leads to

$$
\begin{aligned}
\mathbf{F} \cdot\left(\boldsymbol{d}_{2} \times \mathbf{x}_{2}\right) & =0 \\
\mathbf{F} \cdot\left(\boldsymbol{d}_{2} \times \mathbf{z}_{2}\right) & =0 \\
\mathbf{F} \cdot \mathbf{y}_{2} & =0
\end{aligned} \quad \Longrightarrow \quad \begin{aligned}
& \mathbf{x}_{2} \cdot\left(\mathbf{F} \times \boldsymbol{d}_{2}\right)=0 \\
& \mathbf{z}_{2} \cdot\left(\mathbf{F} \times \boldsymbol{d}_{2}\right)=0
\end{aligned}
$$

which are reduced to

$$
\mathbf{F} \times \boldsymbol{d}_{2}=d_{2} \mathbf{y}_{2}, \quad \mathbf{F} \cdot \mathbf{y}_{2}=0
$$

Substituting (55) into (59) yields

$\boldsymbol{d}_{1} \times \boldsymbol{d}_{2}=d_{1} d_{2} \mathbf{y}_{2}, \quad \mathbf{F} \cdot \mathbf{y}_{2}=0$ 
The first equality implies that the spherical notch must be on the blade plane. And the second equality means that the normal of the blade must be perpendicular to the direction of F. The design is shown in Fig. $7 \mathrm{~b}$.

\subsubsection{Case 3: S-2R}

For this design case, we first recognize that an S-joint provides three rotations (3R). We just need to make up two more translations with two notch hinges (R). We have shown in Sect. 3.2 that two parallel rotations produce a translation and three parallel rotations produce two translations. Therefore we can have two possible designs. One design utilizes two perpendicular R-joints. Each R-joint is parallel to one rotation axis of the S-joint. The direction of the translational constraint is along the direction that is perpendicular to both R-joint axes. See Fig. 7c.

The other design uses two parallel R-joints which together with one rotation of the $S$-joint produce two translations. The S-joint and two R-joints axes are co-planar. The direction of the translational constraint is parallel to the R-join axis. And the constraint line passes through the center of S-joint. See Fig. 7d. Note the functional body cannot translate along the direction indicated with its orientation fixed.

\subsubsection{Case 4: B-B}

The synthesis of B-B follows a similar procedure of the case $\mathrm{S}-\mathrm{B}$. For this case, both blades must satisfy the conditions in Eq. (59), i.e.

$\boldsymbol{d}_{1}=d_{1} \mathbf{y}_{1} \times \mathbf{F}, \quad \mathbf{F} \cdot \mathbf{y}_{1}=0$

$\boldsymbol{d}_{2}=d_{2} \mathbf{y}_{2} \times \mathbf{F}, \quad \mathbf{F} \cdot \mathbf{y}_{2}=0$

where we have applied a vector algebra operation. This means that the normal of both blades must be perpendicular to the direction $\mathbf{F}$. However the two blades must not be parallel to each other as otherwise they would be redundant. The intersection line of the blades is the constraint line. Depending on how the blades are oriented, we can have two possible designs. In the design shown in Fig. 7e, the constraint line is along the longitude direction of the blades. And in the design shown in Fig. 7f, the constraint line is along the width direction of the blades.

\subsubsection{Case 5: B-2R}

Since a blade has two rotations and one translation, we can make up one rotation and one translation with two R-joints. Obviously one R-joint must be along the normal of the blade plane in order to make up the third rotation. The second Rjoint is parallel to the first $\mathrm{R}$-joint. These two parallel R-joints produce one additional translation along the local $\mathrm{z}$-axis of the blade. See Fig. 7g.

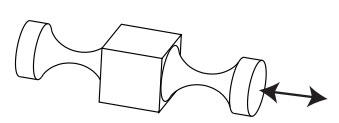

(a)

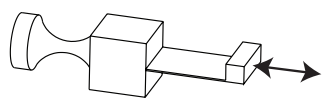

(b)
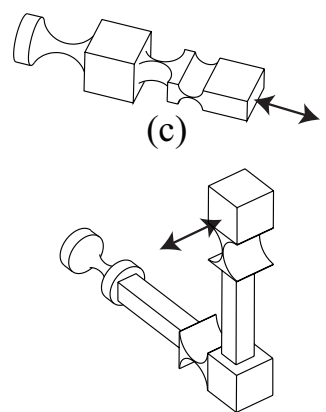

(d)

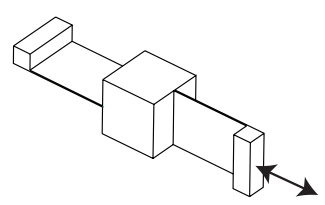

(e)

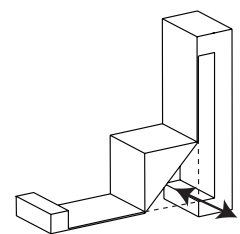

(f)

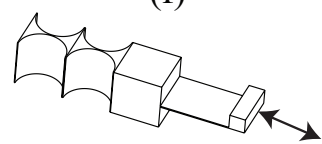

(g)

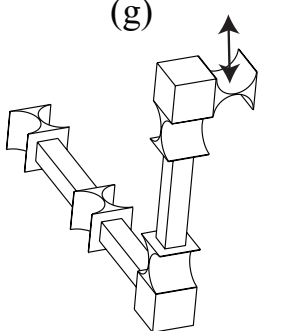

(h)

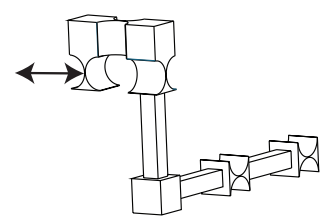

(i)

Figure 7. Various designs of P-constraint $\hat{W}_{P}$ with serial chains of flexure primitives $\mathrm{S}, \mathrm{B}$ and $\mathrm{R}$. The double head arrows represent the direction of the constrained translation. One end of the chains is fixed. (a) case 1 with two spherical notches (2S). (b) case 2 with one spherical notch and one blade (S-B). (c-d) case 3 with one spherical notch and two notch hinges (S-2R). (e-f) case 4 with two intersecting blades (2B). (g) case 5 with one blade and two notch hinges (B-2R). (h-i) case 6 with five notch hinges (5R).

\subsubsection{Case 6: 5R}

Synthesis of this case is simple. To produce two translations with a serial chain of $5 \mathrm{R}$, we can have two possible designs by using parallel rotations. In one design, we have two Rjoints parallel to one plane and another two R-joints parallel to a second plane. This design takes advantage of the fact that two parallel rotations produce a translation as proven previously. The direction of the translational constraint is along. See Fig. 7 h.

And the other design uses three parallel R-joints which produce two translations in the plane normal to the axis of the R-joints. The direction of the translational constraint is along the the axis of the three parallel R-joints. See Fig. 7i.

\subsection{Synthesis of R-Constraints $\hat{W}_{R}$}

A R-constraint element removes a single rotation while allows all other motions. The constraint space of a rotational 
constraint is pure couple wrench $\hat{W}_{R}$ given in (5), which we copy here for convenience

$$
\hat{W}_{R}=\left\{\begin{array}{c}
\mathbf{0} \\
\mathbf{M}
\end{array}\right\}
$$

Our goal is to use serial chains of at least two flexure primitives to design $\hat{W}_{R}$. That is to make up its complementary freedom space which consists of two rotations (R) and three translations $(\mathrm{P})$. Since a bellow spring is itself a rotational constraint, we do not consider it in our design. And wires and spherical notches are also excluded in design as their freedom space already consists of three rotations. Therefore we only consider primitives B and R. There are three potential designs B-B, B-2R and 5R.

\subsubsection{Case 1: B-B}

To synthesize a serial chain of B-B for a rotational constraint, we first write the twist matrix of the blades as

$\left[T_{i}\right]=\left[\begin{array}{ccc}\mathbf{x}_{i} & \mathbf{z}_{i} & \mathbf{0} \\ \boldsymbol{d}_{i} \times \mathbf{x}_{i} & \boldsymbol{d}_{i} \times \mathbf{z}_{i} & \mathbf{y}_{i}\end{array}\right], \quad i=1,2$

They must be reciprocal to $\hat{W}_{R}$ in (63). This leads us the following necessary conditions

$\mathbf{M} \cdot \mathbf{x}_{i}=0, \quad \mathbf{M} \cdot \mathbf{z}_{i}=0$

which implies that

$\mathbf{y}_{1}=\mathbf{y}_{2}=\mathbf{M}$

Therefore, we conclude that two blades must be parallel to each other with their $\mathbf{y}$-(normal) axis along $\mathbf{M}$. The two parallel blades must be separated by a nonzero distance in order to produce sufficient translations in three directions. This design is shown in Fig. 8a.

\subsubsection{Case 2: B-2R}

Tee design of B-2R is synthesized as the following. The B flexure has a freedom space of 2R-P. We have to use two Rjoints to make up two extra translations. And the two translations must be in the blade plane. The only solution is to have one R-joint parallel to the bending direction of the blade and the other R-joint parallel the torsion direction of the blade. And they should be separated by nonzero distance. This design is shown in Fig 8b.

\subsubsection{Case 3: 5R}

This case is trivial as there is only one possible design. That is, three R-joints are parallel to one plane and the other two $\mathrm{R}$-joints parallel to a second plane. And these two planes are perpendicular to each other. See Fig. 8c.

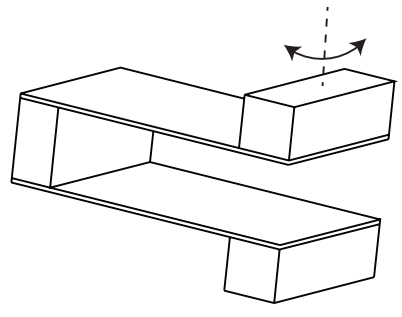

(a)

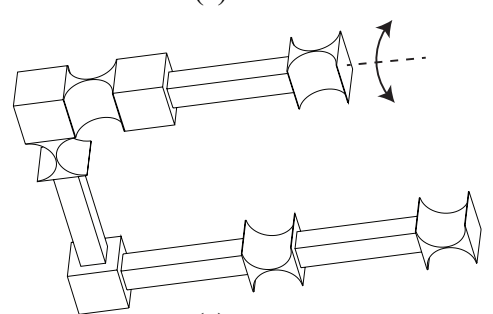

(c)

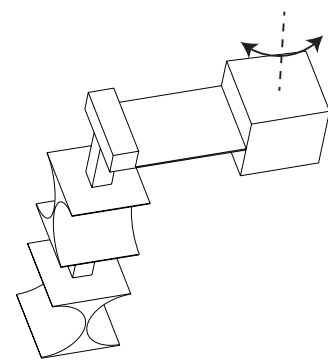

(b)
Figure 8. Various designs of the rotational constraint $\hat{W}_{R}$ with serial chains of flexure primitives $B$ and $R$. The arrowed arcs represent the rotation constrained by the flexure design. One end of the chains is fixed.

\section{Synthesis of hybrid structures}

We can build more complex flexure mechanisms with hybrid structures of flexure primitives together with the freedom and constraint elements synthesized in the previous sections. Here a hybrid structure is a structure with both serial and parallel connections. As a matter of fact, many spatial flexure mechanisms in practice are in hybrid structures (Yao et al., 2008; Dong et al., 2008). In this section, we use four examples to demonstrate how to design hybrid structures.

\subsection{Hybrid designs of joints and constraints}

In the previous sections, we have presented synthesis of R-joints, P-joints, R-constraints, P-constraints with flexure primitives in Table 1. Although these procedures concern designs of notch hinges (R), blades (B), spherical notches (S), long wires $(\mathrm{W})$ and bellow springs $\left(\mathrm{B}_{\mathrm{s}}\right)$, designers should be aware that there may be multiple choices for these primitives. For instance, a blade flexure of any of these designs can be replaced by a rotational symmetric cylinder or a disc coupling since they have identical freedom and constraint space. Similarly, a wire flexure can be substituted with a corner blade and a spherical notch with a short wire, a notch hinge with a short mean or a split tube. Moreover, a notch hinge of any design can be replaced with the R-joint designs listed in Fig. 4.

These designs are by no means the complete list of the basic freedom and constraint elements as many flexure mechanisms are in hybrid structures which are combinations of serial and parallel chains. For instance, Fig. 9a shows the well known parallelogram 4-bar design with four notch hinges. This structure is considered as a hybrid structure that is 


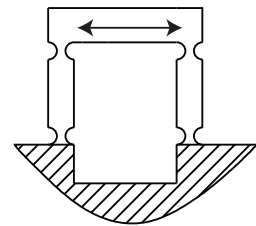

(a)

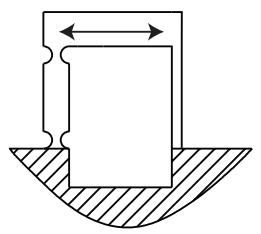

(b)

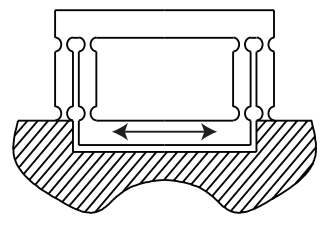

(c)
Figure 9. Designs of P-joints with hybrid structures.

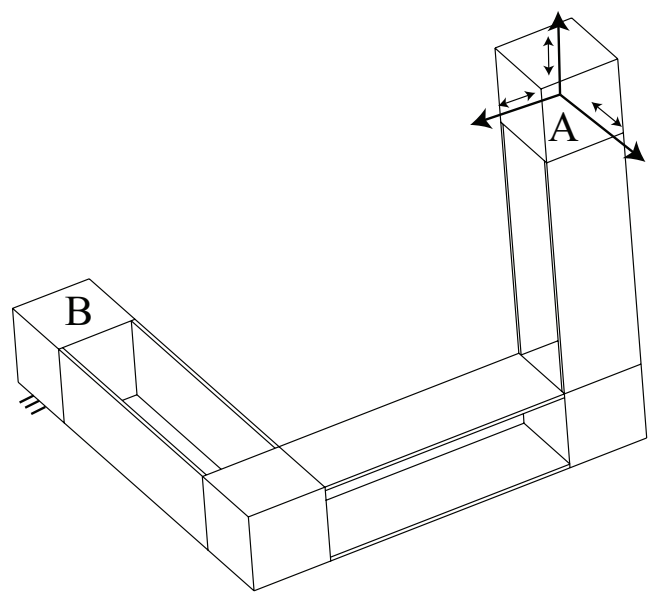

Figure 10. A serial chain of three P-joints connected with two intermediate bodies. Each P-joint is a parallel blade design shown in Fig. 5a. Body B is the fixed base. Body A is the functional body. The arrow lines represent three translations along three coordinate axes.

formed by two identical limbs assembled in parallel. Each limb is a serial chain of two R-joints. It is not hard to prove that this hybrid structure allows only one translation indicated in the figure. If we replace one of the RR chain with a blade flexure, we obtain another design shown in Fig. 9b. And Fig. 9c shows a double parallelogram design that is formed by a serial connection of two parallelogram 4-bar design. This design is widely used to reduce parasitic errors and increase the displacement stroke.

\subsection{Synthesis of serial chains with freedom elements}

As we discussed previously, we can use a serial chain of $\mathrm{R}$-joints and P-joints to construct a freedom space with any combination of DOF. For instance, a serial chain of three Pjoints can make up a freedom space of three translations. If we use the double parallel flexure structure for the P-joint design, we obtain a serial PPP design with three decoupled translations. See Fig. 10. Of course, one can further increase the structural stiffness with assembling multiple serial chains of PPP in parallel (Awtar et al., 2011).

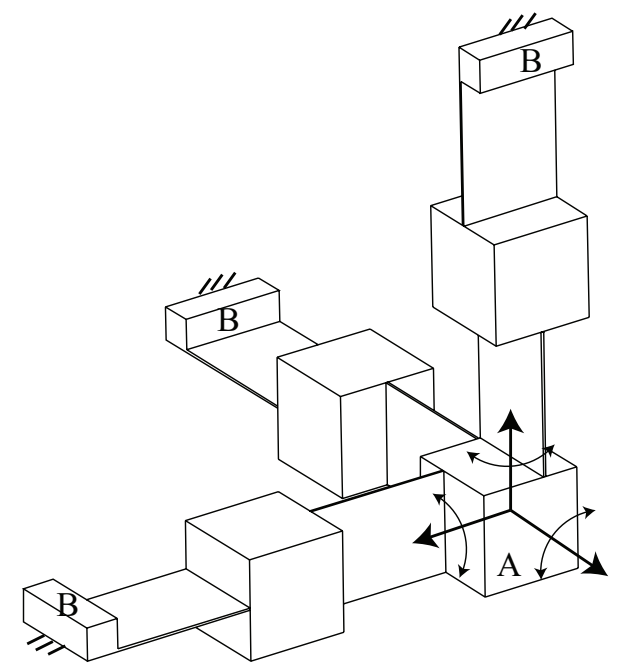

Figure 11. A parallel kinematic chain of three translational constraints results in a design with three rotations about the center of the functional body A. The body B are fixed.

\subsection{Synthesis of parallel chains with constraint elements}

In duality, one can also synthesize parallel chains with the constraint elements synthesized in the previous sections. For instance, if we would like to design a parallel structure with three rotations, we just need to use three translational constraint elements to remove all translations. If we choose the BB design in Fig. 7e for all three translational constraints, we obtain the design shown in Fig. 11. The functional body A can rotate about its center relative to the base body $\mathrm{B}$ while its translations are constrained.

As another example, we would like to design a parallel structure with three translations. We just need to use three rotational constraint elements to remove all rotations. If we choose the BB design in Fig. 8a for all three rotational constraints, we obtain the design shown in Fig. 12. The functional body A can translate in all directions while its rotations are constrained.

\section{Conclusions}

A list of commonly used flexure primitives is first categorized. A complete list of rotational and translational freedom elements is synthesized with parallel connections of these primitives. In duality, translational and rotational constraint elements are synthesized with serial chains of flexure primitives. These freedom and constraints elements form a catalogue of basic building blocks for constructing more complex flexure mechanisms. We have also demonstrate with examples the use of these building blocks with design of hybrid structures for any combinations of freedom. The importance of this work lies in the fact that it formalizes the 


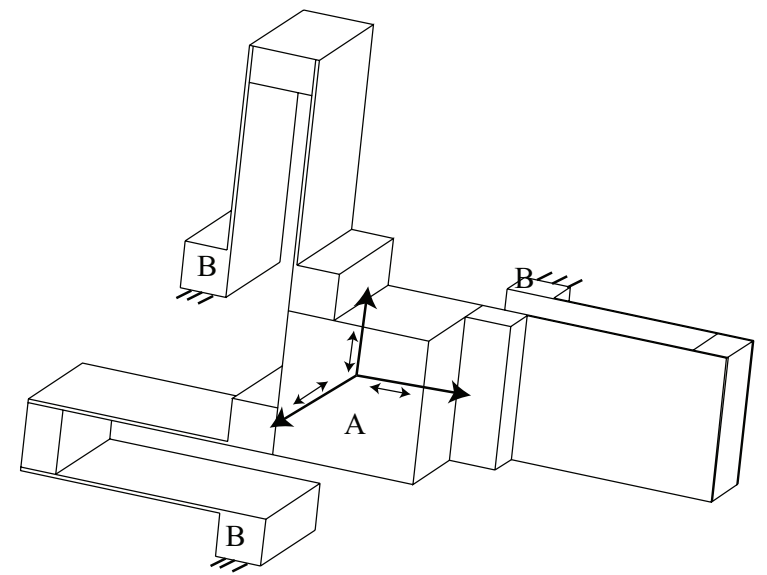

Figure 12. A parallel kinematic chain of three rotational constraints result in a design with three translations along the direction indicated in the figure. The body B are fixed. The body $\mathrm{A}$ is the functional body.

type synthesis (type/toplogy selection) of flexure mechanisms which has been an ad hoc process. It also paves the way towards to design automation and systematic invention of new flexure machinery.

Acknowledgements. This material is based upon work supported by the National Science Foundation under Grant No: CMMI-1161841. Any opinions, findings, and conclusions or recommendations expressed in this material are those of the author(s) and do not necessarily reflect the views of the National Science Foundation.

Edited by: G. Hao

Reviewed by: two anonymous referees

\section{References}

Awtar, S. and Slocum, A. H.: Constraint-based design of parallel kinematic xy flexure mechanisms, J. Mech. Des.-T. ASME, 129, 816-830, 2007.

Awtar, S., Ustick, J., and Sen, S.: An XYZ Parallel Kinematic Flexure MechanismWith Geometrically Decoupled Degrees of Freedom, ASME Journal of Mechanisms and Robotics, 5, 119-126, 2011.

Ball, R. S.: The Theory of Screws, Cambridge University Press, Cambridge, England (Originally published in 1876 and revised by the author in 1900, now reprinted with an introduction by $\mathrm{H}$. Lipkin and J. Duffy), 1998.

Blanding, D. L.: Exact Constraint: Machine Design Using Kinematic Processing, ASME Press, New York, NY, 1999.

Brouwer, D., de Jong, B., and Soemers, H.: Design and modeling of a six DOFs MEMS-based precision manipulator, Precis. Eng., 34, 307-319, 2010.

Chen, S.-C. and Culpepper, M. L.: Design of a six-axis micro-scale nanopositioner- $\mu$ HexFlex, Precis. Eng., 30, 314-324, 2006.
Culpepper, M. L. and Anderson, G.: Design of a low-cost nanomanipulator which utilizes a monolithic, spatial compliant mechanism, Precis. Eng., 28, 469-482, 2004.

Dai, J. S. and Jones, J. R.: Interrelationship between screw systems and corresponding reciprocal systems and applications, Mech. Mach. Theory, 36, 633-651, 2001.

Dai, J. S. and Jones, J. R.: A Linear Algebraic Procedure in Obtaining Reciprocal Screw Systems, J. Robot. Syst., 20, 401-412, 2003.

Davidson, J. K. and Hunt, K. H.: Robots and Screw Theory: Applications of Kinematics and Statics to Robotics, Oxford University Press, New York, NY, 2004.

Dong, J., Yao, Q., and Ferreira, P. M.: A novel parallel-kinematics mechanism for integrated, multi-axis nanopositioning: Part 2: Dynamics, control and performance analysis, Precis. Eng., 32, 20-33, 2008.

Hale, L. C.: Principles and Techniques for Designing Precision Machines, Ph.D. thesis, MIT, Cambridge, MA, 1999.

Henein, S.: Flexures: simply subtle, tutorial on the design of flexuremechanisms, Second International Symposium on Compliant Mechanisms, IFToMM/ASME CoMe 2011, delft, the Netherlands, 2011.

Hopkins, J.: Modeling and Generating New Flexure Constraint Elements, in: Proc. of the 12th EUSPEN International Conference, 424-428, Stockholm, Sweden, 2012.

Hopkins, J. B.: Design of parallel flexure systems via freedom and constraint topologies (FACT), MS, Massachusetts Institute of Technology, Cambridge, MA, 2007a.

Hopkins, J. B.: Design of flexure-based motion stages for mechatronic systems via freedom, actuation and constraint topologies (FACT), Ph.D. thesis, Massachusetts Institute of Technology, Cambridge, MA, 2007b.

Hopkins, J. B. and Culpepper, M. L.: Synthesis of multi-degree of freedom, parallel flexure system concepts via Freedom and Constraint Topology (FACT) - Part I: Principles, Precis. Eng., 34, 259-270, 2010a.

Hopkins, J. B. and Culpepper, M. L.: Synthesis of multi-degree of freedom, parallel flexure system concepts via freedom and constraint topology (FACT). Part II: Practice, Precis. Eng., 34, 271278, 2010b.

Hopkins, J. B. and Culpepper, M. L.: A screw theory basis for quantitative and graphical design tools that define layout of actuators to minimize parasitic errors in parallel flexure systems, Precis. Eng., 34, 767-776, 2010c.

Hopkins, J. B. and Culpepper, M. L.: Synthesis of precision serial flexure systems using freedom and constraint topologies (FACT), Precis. Eng., 35, 638-649, 2011.

Howell, L. L.: Compliant Mechanisms, Wiley-Interscience, New York, NY, 2001.

Huang, Z., Liu, J., and Zeng, D.: A general methodology for mobility analysis of mechanisms based on constraint screw theory, Science in China Series E: Technological Sciences, 52, 1337 1347, 2008.

Hunt, K. H.: Kinematic Geometry of Mechanisms, Oxford University Press, New York, NY, 1978.

Kong, X. and Gosselin, C. M.: Type Synthesis of Parallel Mechanisms, Springer, softcover reprint of hardcover 1st Edn. 2007 Edn., 2010. 
Phillips, J.: Freedom in Machinery. Volume 1, Introducing Screw Theory, Cambridge University Press, Cambridge, UK, 1984.

Phillips, J.: Freedom in Machinery. Volume 2, Screw Theory Exemplified, Cambridge University Press, Cambridge, UK, 1990.

Smith, S. and Chetwynd, D.: Foundations of Ultra-Precision Mechanism Design, CRC Press LLC, 1992.

Smith, S. T.: Flexure: Element of Elastic Mechanisms, CRC Press LLC, London, UK, 2000.

Su, H.-J.: Mobility Analysis of Flexure Mechanisms via Screw Algebra, ASME Journal of Mechanisms and Robotics, 3, 041010, doi:10.1115/1.4004910, 2011.

Su, H.-J. and Tari, H.: Realizing Orthogonal Motions With Wire Flexures Connected in Parallel, J. Mech. Des.-T. ASME , 132, 121002, doi:10.1115/1.4002837, 2010.

Su, H.-J. and Tari, H.: On Line Screw Systems and Their Application to Flexure Synthesis, ASME Journal of Mechanisms and Robotics, 3, 011009, doi:10.1115/1.4003078, 2011.

Su, H.-J., Dorozhkin, D. V., and Vance, J. M.: A Screw Theory Approach for the Conceptual Design of Flexible Joints for Compliant Mechanisms, ASME Journal of Mechanisms and Robotics, 1, $041009.1-041009.8,2009$.
Varadarajan, K. M. and Culpepper, M. L.: A dual-purpose positioner-fixture for precision six-axis positioning and precision fixturing: Part I. Modeling and design, Precis. Eng., 31, 276-286, 2007a.

Varadarajan, K. M. and Culpepper, M. L.: A dual-purpose positioner-fixture for precision six-axis positioning and precision fixturing: Part II. Characterization and calibration, Precis. Eng., 31, 287-292, 2007b.

Yao, Q., Dong, J., and Ferreira, P. M.: A novel parallel-kinematics mechanisms for integrated, multi-axis nanopositioning: Part 1. Kinematics and design for fabrication, Precis. Eng., 32, 7-19, 2008.

Yu, J. J., Li, S. Z., Pei, X., Su, H.-j., Hopkins, J. B., and Culpepper, M. L.: Type Synthesis Principle and Practice of Flexure Systems in the Framework of Screw Theory: Part I - General Methodology, Proceedings of the ASME 2010 International Design Engineering Technical Conferences and Computers and Information in Engineering Conference, 543-552, doi:10.1115/DETC201028783, 2010. 